\title{
Berücksichtigung des Empowerments in der Programmplanung von Gesundheitsförderung
}

\section{Empowerment and Health Promotion Programming}

\section{Autor}

Institut

\section{G. Laverack}

University of Auckland, Neuseeland

\author{
Schlüsselwörter \\ - Gesundheitsförderung \\ - Programmplanung \\ - Empowerment \\ - Partizipation \\ - Gemeinde
}

\section{Key words}

- health promotion

- programme planning

- empowerment

- participation

- community

\section{Bibliografie}

DOI 10.1055/s-0028-1103259

Gesundheitswesen 2008;

70: 736-741

(c) Georg Thieme Verlag KG

Stuttgart · New York

ISSN 0941-3790

\section{Korrespondenzadresse}

\section{Dr. G. Laverack}

Director of Health Promotion School of Population Health

The University of Auckland

Auckland, Neuseeland

g.laverack@auckland.ac.nz

\section{Zusammenfassung \\ $\nabla$}

Bei gesundheitsförderlichen Programmen kommt es oft zu Spannungen zwischen dem so genannten „Bottom-up“- und dem „Top-down“-Ansatz. Der „Bottom-up“-Ansatz ist mit Übertragung von Verantwortung auf die Gemeinde und damit dem Empowerment der Gemeinde verbunden. Es werden zunächst die Themen aufgegriffen, die für bestimmte Gruppen oder Personen in der Gemeinde wichtig sind. Die Ausweitung von Kontrolle auf Gemeindemitglieder wird als wichtiger Bestandteil des gesundheitlichen Nutzens betrachtet. Der „Top-down“-Ansatz zielt hingegen auf die Prävention von Krankheiten ab und beginnt damit, dass die Zielgruppe des Programms an Themen herangeführt wird, die zuvor „von oben“, z.B. durch Gesundheitsbehörden, festgelegt wurden. Verbesserungen des gesundheitsbewussten Verhalten oder von biomedizinischen Parametern gelten hier als wichtigste Ergebnisse. Ein Empowerment der Gemeinde wird nur als Mittel zum Zweck der gesundheitsbezogenen Verhaltensänderung betrachtet. Der Konflikt zwischen diesen beiden Ansätzen ist jedoch nicht unlösbar. Dieser Artikel stellt ein Rahmenprogramm vor, das sog. „parallel-tracking“ (Verfolgen paralleler Pfade), das Gesundheitsförderern helfen soll, die Ziele des gemeindebezogenen Empowerments systematisch in „Top-down“Gesundheitsprogramme zu integrieren.

\section{Abstract \\ $\nabla$}

Health promotion often presents a tension between "bottom up" and "top down" programming. "Bottom-up" is associated with community empowerment and begins on issues of concern to particular groups or individuals and regards an increase in overall control as an important element of the health outcome. "Top-down" is associated with disease prevention efforts and begins by seeking to involve beneficiaries on issues defined by health agencies. It regards improvements in health behaviours or bio-medical indicators as the important outcome and community empowerment is viewed simply as a means to the end of health behaviour change. The tension between these two approaches is not unresolvable, and this article presents a framework, the "parallel-track", intended to assist health promotion practitioners to systematically accommodate community empowerment goals within "top-down" health programming.
Übersetzung aus dem Englischen und Bearbeitung: PD Dr. Julika Loss, Franziska Gerner, Institut für Medizinmanagement und Gesundheitswissenschaften, Universität Bayreuth, 95440 Bayreuth. 


\section{Einleitung}

\section{Programmplanung in der Gesundheitsförderung}

Gesundheitförderung wird häufig durch die Regierung, Gesundheitsbehörden, Krankenkassen oder den öffentlichen Gesundheitsdienst geplant und gesteuert. Diejenigen, die in der Gesundheitsförderung arbeiten, z.B. Projektleiter/innen ${ }^{1}$, sind oft im öffentlichen Gesundheitsdienst angestellt und haben die Aufgabe, für die Bereitstellung und Weiterleitung von Informationen, Mitteln und Dienstleistungen an einzelne Personen, Gruppen oder Gemeinden zu sorgen. Die Informationen bzw. Maßnahmen richten sich dabei an verschiedene Zielgruppen (zum Beispiel Jugendliche, schwangere Frauen, übergewichtige Männer mittleren Alters), um ihre Gesundheit zu fördern und ihre Lebensqualität zu verbessern. In der Praxis wird Gesundheitsförderung meistens durch eine Kombination von verschiedenen Aktivitäten im Rahmen eines Projekts, einer Intervention oder Programms durchgeführt; man kann von Programmplanung in der Gesundheitsförderung sprechen. Die Programmplanung liegt in der Regel beim Programmleiter und beeinhaltet Phasen der Forschung und Bedarfsermittlung, Konzipierung, Implementierung, Aufrechterhaltung und Evaluation.

Gesundheitsförderungsprogramme werden meist professionell geplant und geleitet. Die Programmleiter oder ihre Auftraggeber bestimmen die Gestaltung, die Art der Implementierung und der Evaluation des Programms. Dies beinhaltet auch die Auswahl von Zielgruppen, von Strategien, mit denen sie erreicht werden sollen, und die Allokation von Ressourcen. Die Initiierung des Programms und das Engagement bei der Umsetzung hängen ebenso vom Programmleiter ab. Er befindet sich somit in einer relativ einflussreichen Position gegenüber den Personen, für die das Programm entwickelt wurde [1].

Eine der grundlegenden Fragen bei der Programmplanung ist, durch welche Vorgehensweise die gesundheitlichen Anliegen angegangen werden sollen. Dies kann auf zwei verschiedenen Wegen geschehen: über „Top-down“- oder „Bottom-up“-Ansätze. Der „Top-down“-Ansatz beschreibt Programme, bei denen das Ziel der Maßnahme durch vorgesetzte Strukturen bzw. Autoritäten definiert wird (top), z.B. die Steigerung von körperlicher Aktivität, und an die Zielgruppe, z.B. eine Gemeinde ${ }^{2}$, weitergeleitet wird (down). Der „Bottom-up“-Ansatz meint genau das Gegenteil - die Gemeinde oder eine bestimmte Gruppe identifiziert ihre Probleme selbst und teilt diese dann den Entscheidungsträgern, z.B. der jeweils zuständigen Behörde, mit, z.B. asoziales Verhalten von Jugendlichen in örtlichen Parkanlagen. Diese zwei Ausdrücke („Top-down“- und „Bottom-up“-Ansatz) unterstreichen sehr gut die Machtverhältnisse, die in Gesundheitsförderungsprogrammen existieren. Zu den „Topdown“-orientierten Programmen gehören fast alle Maßnahmen

\footnotetext{
${ }^{1}$ Zur Vereinfachung des Leseflusses wird, wenn eine Gruppe weibliche und männliche Personen beinhaltet, die männliche Form gewählt. Hierbei sind immer weibliche und mänliche Personen gleichermaßen gemeint.

${ }^{2}$ Im englischen Original wird für die angestrebten Nutznießer des Programms durchgängig der Begriff der „community“ gewählt, der schwer ins Deutsche übersetzbar ist. Zum einen kann damit eine lokal begrenzte Gemeinde gemeint sein, z.B. ein Dorf oder eine Kommune. Zum anderen kann sich „community“ auch auf eine Gruppe von Personen beziehen, die bestimmte Lebensgewohnheiten, Sprachen o.ä. teilen, z. B. eine Gruppe von Griechisch sprechenden Personen innerhalb einer Stadt. Der hier verwendete Begriff „Gemeinde“ ist in diesem doppelten Sinne zu verstehen. Um die Mehrdeutigkeit des „community“-Begriffs zu erfassen, wird zudem in der deutschen Übersetzung wiederholt von „Gemeinde oder Gruppe“ bzw. „Gemeinde oder Zielgruppe“ gesprochen.
}

der Gesundheitsaufklärung und -erziehung sowie Interventionen, die auf den individuellen Lebensstil und gesundheitsbezogenes Verhalten abzielen. „Top-down“-Ansätze sind die bei weitem vorherrschende Vorgehensweise bei Gesundheitsförderungsprogrammen. „Bottom-up“-orientierte Programme sind seltener; gelegentlich stellen sie ein eigenes Unterprojekt im Rahmen von umfassenden „Top-down“-orientierten Programmen dar [2].

\section{Empowerment in der Gemeinde}

Gemeindebezogenes Empowerment kann als ein Prozess angesehen werden, der es Menschen ermöglicht, mehr Kontrolle über wichtige Themen zu gewinnen, die ihr Leben und ihre Gesundheit beinflussen. Das beinhaltet auch, dass Gruppen oder Gemeinden nach und nach organisiert und mobilisiert werden, um selbst politisch und sozial aktiv zu werden [3]. Der Empowerment-Ansatz ist mittlerweile zu einer recht weit verbreiteten Strategie in der Gesundheitsförderung geworden, indem - zumindest in der Theorie - vor der Umsetzung eines Programmes die Anliegen, die den Zielpersonen wichtig sind, durch Diskussionen, Bedürfniserhebungen etc. identifiziert und dann gezielt durch das Programm thematisiert werden. Wie bereits erwähnt kann das, was in der Praxis der Gesundheitsförderung geschieht, erheblich von dieser Theorie abweichen, was wiederum zu Spannungen führen kann, die letztendlich auf eine ungleiche bzw. paternalistische Beziehung zwischen dem Programmleiter und den Personen aus der Zielgruppe hinauslaufen.

Derartige Spannungen zeigen sich bei vielen gesundheitsförderlichen Programmen, sowohl bei „Top-down“- als auch bei „Bottom-up“-Vorgehensweisen. Im einen Fall nutzt der Programmleiter seine dominante Stellung, um einen vorher definierten Programmablauf bei der Zielgruppe durchzusetzen im Sinne einer „vertikalen“ Programmgestaltung. Im anderen Fall versucht eine Gruppe oder Gemeinde, ihre aktuellen Anliegen auf die Tagesordnung zu setzen, die von denen, die der Programmleiter als wichtig identifiziert hat, durchaus erheblich abweichen können. Sowohl ein externer Programmleiter als auch die Zielgruppe oder Gemeinde haben bestimmte Anliegen oder Bedürfnisse, die sie in einem gesundheitsförderlichen Programm gerne aufgreifen würden. Die Prioritäten der Programmleiter leiten sich normalerweise aus epidemiologischen Daten ab, zum Beispiel die Häufigkeit von Übergewicht bei Schulkindern. Die Prioritäten der Gemeinde leiten sich hingegen typischerweise ab aus:

- unmittelbaren Bedürfnissen, z.B. die Bereitstellung von Kinderbetreuungsplätzen, und/oder

- Problemen, die bereits länger existieren, z.B. Verärgerung und Verunsicherung von Innenstadt-Bewohnern über kaputte Straßenbeleuchtung oder unzureichende Präsenz der Polizei in der Gegend.

Manchmal sind die Anliegen des Programmleiters und die der Zielgruppe oder Gemeinde ähnlich und können bei der Ausrichtung des Programms in Einklang gebracht werden. Häufiger sind die Prioritäten des Programmleiters und die der Zielgruppe aber grundlegend verschieden, und es muss ein Kompromiss gefunden werden. Diese Situation führt normalerweise dazu, dass die Bedürfnisse der Gemeinde im Design eines „Top-down“-Programms nicht berücksichtigt werden. Die Herausforderung für die Programmplanung ist es, den Ansatz des Empowerment einer Gruppe oder Gemeinde erfolgreich in die vorherrschende „Top-down“-Orientierung von Gesundheitsförderung zu integrieren. 


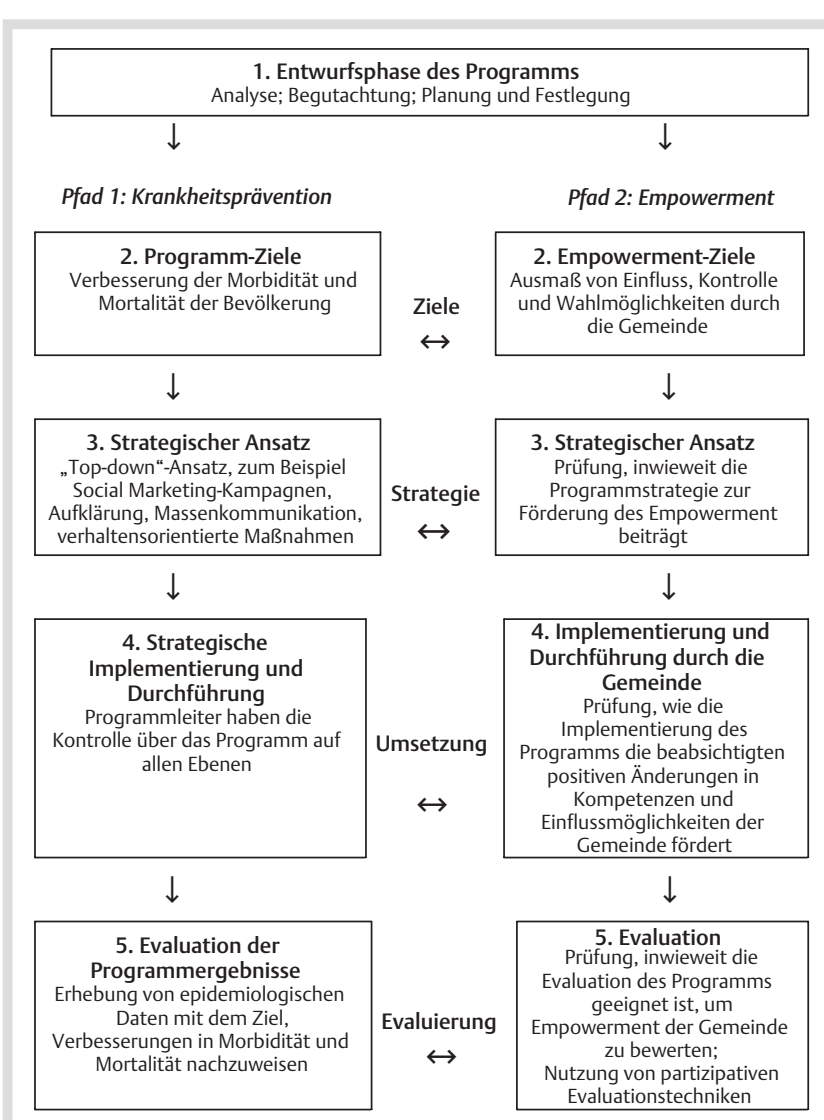

Abb. 1 „Parallel-Tracking“: Berücksichtigung von Empowerment in klassischen „Top-down“ Programmen für chronische Krankheiten.

\section{Der Ansatz des „parallel tracking“}

Um die epidemiologisch gestützten Vorgaben „von oben“ und die aktuellen Bedürfnisse „,von unten“ zu harmonisieren, ist es hilfreich, „Top-down“- und „Bottom-up“-Ansätze nicht als eine einander ausschließende Dichotomie zu sehen, sondern vielmehr die Möglichkeit eines parallelen Prozesses beider Vorgehensweisen innerhalb eines Programmes zu erwägen. Die Integrierung von Methoden zum Gemeinde-Empowerment kann als Vorgehen gesehen werden, das begleitend zur hauptsächlichen, „Top-down“-orientierten Programmumsetzung implementiert wird (sog. „parallel-tracking“, Verfolgen paralleler Pfade, s. ○ Abb. 1). Die Konflikte, die sich naturgemäß zwischen den beiden Arten der Programmgestaltung ergeben, entstehen schrittweise und können so wesentlich besser gelöst werden. In der Praxis erlaubt dieses Vorgehen eine systematische Integration von beiden Stilen der Programmplanung. Das Hauptziel des Gesundheitsprogramms bleibt unverändert, mit einem Fokus auf klassischen „Top-down“-Themen wie z. B. der Reduktion von Krankheitshäufigkeiten. Das gewährleistet, dass die generelle Ausrichtung des Programms mit den Erwartungen der Regierung bzw. der Gesundheitsbehörden übereinstimmt und deswegen auf dieser Ebene eine bessere Akzeptanz findet. Gleichzeitig wird dem Empowerment der Gemeinde aber eine feste, klar umrissene Rolle in dem Programm zugestanden. Wo Empowerment-orientierte Ansätze bereits als Programmbestandteil zum Einsatz kommen, können sie durch „parallel-tracking“ systematisch verstärkt und ausgebaut werden. Wo sie noch nicht im Programmdesign existieren, kann die Struktur des „parallel-tra- cking“ den Programmleiter darauf aufmerksam machen, an welcher Stelle des Ablaufes sie mit zu berücksichtigen sind [2].

Im Folgenden wird eine stilisierte Fallstudie vorgestellt, die darlegt, wie „parallel-tracking“ benutzt werden kann, um Empowerment-Ansätze in jede Phase eines beispielhaften Programms zu integrieren, das auf Menschen mit metabolischem Syndrom zugeschnitten ist.

\section{Die Einbindung von Empowerment-Ansätzen in Pro- gramme zur Prävention von chronischen Krankheiten $\checkmark$ \\ Programme zur Prävention von chronischen Krankheiten}

Programme zur Prävention von chronischen Krankheiten sollen typischerweise eine Veränderung des Lebenstils und des Verhaltens herbeiführen und konzentrieren sich zum Beispiel auf Herzund Kreislauf-Erkrankungen, Diabetes mellitus Typ 2, Übergewicht oder Tabakkonsum. Hier wird das Beispiel des metabolischen Syndroms gewählt. Das metabolische Syndrom besteht aus mehreren, gleichzeitig existierenden Stoffwechselstörungen einschließliche Übergewicht, Bluthochdruck, Diabetes mellitus Typ 2 und Hypercholesterinämie. Es betrifft mindestens jeden fünften Übergewichtigen und ist mit erhöhter Morbidität und Mortalität durch Herz-Kreislauf- und Nierenerkrankungen assoziiert.

Gesundheitsförderungsprogramme in diesem Kontext zielen darauf ab, Menschen mit einem erhöhten Risiko für das metabolische Syndrom dabei zu unterstützen, ihren Lebensstil und ihr Verhalten in Bezug auf Ernährungsgewohnheiten und körperliche Betätigung zu ändern. Übergewicht ist auch für Deutschland ein relevantes Public Health-Thema: Die aktuelle Nationale Verzehrsstudie zeigt, dass in Deutschland 66\% der Männer und $50,6 \%$ der Frauen übergewichtig oder adipös sind (BMI >= $\left.25 \mathrm{~kg} / \mathrm{m}^{2}\right)[4]$.

\section{Die Planungsphase des Programms}

Kernfrage: Wer verfügt über die Mittel und entscheidet über die Implementierung und Leitung des Programms?

In der Planungsphase wird die Machtbeziehung zwischen dem Programmleiter bzw. seinem Auftraggeber und den vorgesehenen Nutznießern des Programms festgelegt. „Top-down“-Programme sind typischerweise geprägt von hierarchischen Strukturen, wobei der Programmleiter in der Regel über die finanziellen Mittel und andere Ressourcen des Programms verfügen kann.

Der Ansatz des „parallel-tracking“ verschiebt die Machtverhältnisse, da der Programmleiter sich in einer Konstellation wiederfindet, in der Verfügungsgewalt und Kontrolle von allen geteilt werden. Die Rolle des Programmleiters ist es nun auch, die Zielgruppe bzw. Gemeinde dabei zu unterstützen, Einfluss zu nehmen und Determinanten, die ihr Leben und ihre Gesundheit bestimmen, selbst zu beeinflussen. In der Planungsphase des Programms nimmt der Programmleiter zwei wesentliche Aufgaben zur Förderung des Empowerments wahr:

- Der Programmleiter unterstützt die Gemeinde- bzw. Gruppenmitglieder dabei, sich aktiv einzubringen und im Laufe des Programms ihre Probleme durch eigene Aktivitäten zu lösen.

- Der Programmleiter hilft Gemeindemitgliedern dabei, diejenigen Themen und Bedürfnisse zu identifizieren, die besonders relevant sind und die durch das Programm berücksich- 
tigt werden sollten, und diese in eine Reihenfolge zu bringen. Eine einfache standardisierte Umfrage oder Beratung durch einzelne Gemeindemitglieder oder Interessengruppen reicht dazu in der Regel nicht aus.

Das Ziel dabei ist, aktiv mit der Gemeinde oder ihren Vertretern in Kontakt zu treten und ihnen eine Stimme zu verleihen, so dass sie ihre Sorgen und Bedürfnisse selbst ausdrücken können. Das kann auf einer allgemeinen Basis geschehen (z. B. „Was sind Ihre Anliegen im Bezug auf Gesundheit?“), oder spezifisch auf das Programm bezogen sein (z. B. „Was sind Ihre Anliegen im Bezug auf Herzkrankheiten?"). Bezogen auf das Beispiel von chronischen Erkrankungen bzw. dem metabolischen Syndrom können die Anliegen der Gemeinde z.B. beeinhalten:

- Bessere Sportanlagen, die gut zugänglich und finanziell erschwinglich sind und so ausgebaut, dass körperliche Bewegung attraktiv wird

- Spezielle Wander- oder Lauf-Gruppen für ältere Menschen, auch als Mittel, um soziale Kontakte zu knüpfen

- Mehr Information über Diabetes mellitus, die über verschiedene Kommunikationskanäle der Gemeinde verfügbar sind

Auf dem „Präventionspfad“ wird parallel dazu festgelegt, welche Präventions-Ziele das Programm erreichen soll. In Bezug auf das metabolische Syndrom würden diese Ziele z. B. beeinhalten:

- Reduzierung von Übergewicht, gemessen durch den Body Mass Index

- Verringerung von Bluthochdruck

- Verringerung von Fettstoffwechselstörungen (Hypercholesterinämie)

Der entscheidende Punkt bei der Programmplanung ist nun, wie der „Präventionspfad“ (festgelegt durch die Autorität von außen) während der verschiedenen Schritte des Programms mit dem Empowermentpfad (bestimmt durch die Gemeinde) verknüpft werden kann. Ziel des Programms muss es sein, die Präventionsziele zu erreichen und gleichzeitig Empowerment auf Gemeindeebene aufzubauen. Wie die finanziellen, materiellen, personellen und intellektuellen Ressourcen, die dem Programm zur Verfügung stehen, verteilt werden, um beiden Zielsetzungen nachzukommen, muss in Verhandlung mit den Gemeinde- bzw. Gruppenmitgliedern ausgehandelt werden.

Bei der Planung des Programms muss auch berücksichtigt werden, dass bei der Zielgruppe oder Gemeinde anfangs die notwendigen Fähigkeiten und Kompetenzen wenig entwickelt sind. Daher sollten in dieser ersten Phase des Programms bereits Strategien verankert werden, um die Kenntnisse und Qualifikation zu vermitteln, die in der Zielgruppe erforderlich sind, wenn sie tatsächlich mehr Einfluss auf die Programmgestaltung ausüben soll.

Wie lange es dauert, bis eine Gemeinde oder Gruppe sich diese Kompetenzen angeeignet hat, hängt von den individuellen Fähigkeiten sowie der Leistungsfähigkeit der gesamten Gruppe ab. Hat ein Programm zur Gesundheitsförderung einen zu engen Zeitrahmen, so besteht das Risiko, dass Empowerment- und Kompetenz-bezogene Veränderungen auf Ebene der Gemeinde initiiert werden, die Unterstützung aber endet, bevor die angestrebten Fähigkeiten und Qualifikationen erreicht wurden.

\section{Festlegen der Ziele des Programms}

Kernfrage: Wie werden die Programmziele zur Prävention und die Empowermentziele in der Programmgestaltung in Einklang gebracht?

Die Zielsetzung in Präventionsprogrammen für chronische Krankheiten konzentriert sich normalerweise auf die Reduktion der Morbidität und Mortalität und auf die Veränderung von Lebensstil und gesundheitsbezogenem Verhalten. Die Zielsetzung im Empowerment konzentriert sich hingegen auf das Ausmaß der Entscheidungsmacht, die die Gemeinde in Bezug auf Determinanten hat, die ihre Gesundheit beeinflussen. Die spezifische Art der Präventionsziele wird je nach inhaltlicher Ausrichtung des Programms variieren. Der Zweck des „parallel-tracking“ ist es, Empowermentziele zu entwickeln, die die Präventionsziele ergänzen.

Die Empowermentziele basieren auf den Bedürfnissen und Anliegen der Gemeinde. Die Rolle des Programmleiters ist es, den Gemeindemitgliedern dabei zu helfen, Ziele zu formulieren, die klar und erreichbar, realistisch und messbar sind. Weiterhin ist er dafür verantwortlich, dass spezifische Aktivitäten aufgezeigt werden, die zur Erreichung der Ziele notwendig sind, und diese Aktivitäten in eine sinnvolle Reihenfolge gebracht werden. $\mathrm{Zu}$ dem sollte er Gemeindemitglieder bei der Festlegung von konkreten Aufgaben und individuellen Verantwortlichen unterstützen, um einen erfolgreichen Ablauf zu gewährleisten. Dazu gehört auch das Festlegen eines realistischen Zeitrahmens sowie sinnvoller Indikatoren.

Am Beispiel eines Präventionsprogramms für das metabolische Syndrom könnten die Empowermentziele folgendes enthalten:

- Unterstützung der Gemeinde, zwei geeignete Sportanlagen in ihrer näheren Umgebung ausfindig zu machen und zu nutzen - bis zum Ende des Jahres 2008.

- Unterstützung der Gemeinde bei der Gründung von fünf Walkinggruppen für Männer und Frauen bis zum Ende des Jahres 2008.

- Die Durchführung von 30 Seminaren und Diskussionsveranstaltungen zum Thema Diabetes mellitus an verschiedenen Veranstaltungsorten bis zum Ende des Jahres 2008, mit der Möglichkeit der Weiterverweisung von Personen, die einen weiteren Informationsbedarf haben.

Die Empowermentziele müssen flexibel sein, da es sehr wahrscheinlich ist, dass sie sich mit der wachsenden Erfahrung der Gemeindemitglieder im Laufe der Zeit ändern. Dieser Prozess kann durch Diskussion und Problemanalyse mit Hilfe des Programmleiters unterstützt werden und hilft der Gruppe dabei, sich auf unmittelbare und lösbare Probleme zu fokussieren, wie zum Beispiel bessere Zugangsmöglichkeiten der Sportanlagen für Behinderte.

Die Präventionsziele müssen ebenfalls klar und erreichbar sein, zum Beispiel:

- Reduktion des Body Mass Index um 10\% bei $50 \%$ der untersuchten Bevölkerungsgruppe bis Ende 2008.

- Das Erreichen von normalen Blutdruckwerten bei 50\% der untersuchten Bevölkerungsgruppe bis Ende 2008.

- Das Erreichen von normalen Cholesterinwerten bei 50\% der untersuchten Bevölkerungsgruppe bis Ende 2008.

\section{Entwicklung des strategischen Ansatzes}

Kernfrage: Wie ist der strategische Ansatz des Präventionspogramms mit dem strategischen Ansatz für Empowerment verknüpft und unterstützt diesen?

Eine Verknüpfung der strategischen Ansätze beider „Pfade“ kann erreicht werden, indem die Stärken und die Eigenschaften der Zielgruppe bzw. Gemeinde (sog. „assets“) in die Programmgestaltung integriert werden. Insbesondere geht es dabei um die Bereiche, die der Zielgruppe erlauben, sich durch soziale und politische Veränderung selbständig zu organisieren und zu mobilisieren hin zu einem Empowerment auf Gemeindeebene. Bei Em- 
Tab. 1 Beispiele der Kompetenzen, die bei den Gemeindemitgliedern im Rahmen des Empowerment-Pfades gefördert bzw. ausgebildet werden sollten.

\begin{tabular}{|ll}
\hline Programmphase & Ausbildung von Fähigkeiten \\
\hline 1. Planung & - systematische Literaturrecherche \\
& - Analyse von epidemiologischen Daten \\
& - Identifizierung der Bedürfnisse der Gemeinde \\
& - Bewertung des Programmplans \\
& - Entwicklung von Zielen, die den SMART-Kriterien entsprechen (spezifisch, messbar, angemessen, relevant, terminiert) \\
\hline 2. Festlegung der Ziele & - Entwicklung eines strukturierten Arbeits- und Zeitplans \\
\hline 3. strategischer Ansatz & - Strategien zur Ermutigung und Befähigung von Individuen, Gruppen und der Gemeinde als Ganzes \\
& - Entwicklung von Materialen \\
& - Theorien und Modelle der Gesundheitsförderung \\
& - interpersonelle Kommunikation \\
& - Moderation von Workshops \\
& - Durchführung von effektiven Gruppentreffen und öffentlichen Vorträgen \\
& - Mittelbeschaffung (Fundraising) und Gewinnen von Ressourcen \\
& - Entwicklung eines Kostenplans \\
4. Implementierung des & - Konfliktmanagement \\
Programmes & - Personalmanagement \\
& - Umgang mit Empfehlungen \\
& - partizipative Erhebungstechniken \\
& - qualitative Forschungsmethoden \\
& - quantitative Forschungsmethoden \\
*s. Laverack G, „Messung, Bewertung und strategische Weiterentwicklung von Gemeindekapazität und -empowerment: Vorstellen eines qualitativen Instruments“ in dieser \\
Ausgabe
\end{tabular}

powerment auf Gemeindeebene sowie Gemeinde-Kapazität („capacity“) handelt es sich um zwei überlappende Prozesse, die grundlegende Formen sozialer Organisation und Mobilisation darstellen und versuchen, Ungleichheiten im Leben der Menschen zu überwinden [3]. Die Gemeindekapazität setzt sich aus neun Dimensionen zusammen:

- Verbesserung der aktiven Teilhabe der Bürger

- Entwicklung von lokalen Führungsqualitäten (sog. „Leadership“)

- Ausbildung von organisatorischen Strukturen, die Empowerment fördern

- Fähigkeit zur Problemanalyse

- Ermutigung zu einem kritischen Bewusstsein

- Verbesserung der Mobilisierung von Ressourcen

- Vernetzung mit anderen Organisationen und Akteuren

- Ausbildung einer gleichberechtigten Beziehung zu Auftraggebern und Experten

- Erhöhter Einfluss auf die Programmdurchführung

Eine ausführlichere Darstellung dieser Kapazitätsdimensionen findet sich bei Laverack G, „Messung, Bewertung und strategische Weiterentwicklung von Gemeindekapazität und -empowerment: Vorstellen eines qualitativen Instruments“ in dieser Ausgabe (S. 764). Die geschilderte Entwicklung der Gemeindekapazität soll als Teil des „parallel-trackings“ in der strategischen Ansatz-Phase des Programms zum Einsatz kommen.

\section{Implementierung des Programms}

Kernfrage: Wie fördert die Implementierung des Präventionspfads den Aufbau von Entscheidungsmacht und Einflussnahme durch die Gemeinde?

Programme zur Gesundheitsförderung werden typischerweise von Experten geleitet. Zu den Aufgaben der Programmverantwortung gehört es traditionell, die Planung, Organisation und Implementierung von Maßnahmen zur Erreichung der Präventionsziele zu überwachen und leiten. Dabei soll der Erfolg des Programms im Sinne von Effektivität (Ausmaß der Zielerrei- chung) und Effizienz (Effektivität im Vergleich mit anderen Möglichkeiten der Zielerreichung) sichergestellt werden [5].

Bei „Bottom-up“-Ansätzen, die auf Empowerment der Gemeinde abzielen, ist es die Aufgabe der Projektleitung, dem Engagement der Gemeinde gegenüber offen zu sein und es zu einer motivierenden Erfahrung für die Gemeindemitglieder zu machen. Das kann dadurch erreicht werden, dass die Zielgruppe bzw. Gemeinde ermutigt wird, vermehrt Verantwortung für die Programmleitung zu übernehmen und dass Fähigkeiten und Kompetenzen gefördert werden, die es den Menschen ermöglichen, an Tätigkeiten wie Berichterstattung, Budgetierung und Evalutation teilzunehmen. $\bullet$ Tab. 1 zeigt einen Überblick über Beispiele einer solchen Kompetenzförderung, die in jeder Stufe des Gesundheitsförderungsprogrammes umgesetzt werden kann.

Ein anderer Weg, um die Fähigkeiten bei der Zielgruppe auszubauen, ist ihre Einbindung in kurzfristige Aufgaben, die im Rahmen des Projekts anfallen und deren Umsetzung realistisch und erreichbar ist. Das ist wichtig, weil kurzfristige Erfolge dabei helfen können, Menschen für längerfristige Ziele zu motivieren. Der Fortschritt sollte regelmäßig mit der Gemeinde begutachtet und bewertet werden, um ihre Erfolge und Misserfolge reflektieren zu können [6]. Am Beispiel des Präventionsprogrammes für das metabolische Syndrom könnte das bedeuten, dass man den Teilnehmern die Fähigkeiten vermittelt, selbst aktiv zu werden, um eigenständig monatliche Walking-Gruppen zu gründen. Im weiteren Verlauf kann man sie dabei unterstützen, regelmäßige wöchentliche Walking-Gruppen einzuführen, für deren Betreuung und Umsetzung sie verantwortlich sind.

Das Anliegen der Programmleitung sollte es sein, der Gemeinde nach und nach mehr Kontrolle über die Verantwortlichkeiten zu geben, die bei der Implementierung des Programms anfallen. Dies setzt voraus, dass das Programmdesign eine Strategie beeinhaltet, die Kompetenz, Fähigkeiten und Kapazitäten der Gemeinde systematisch entwickelt, damit die Gemeindemitglieder ausreichend Qualifikationen und Selbstvertrauen besitzen, um ihre Stärke und Vitalität zu entfalten. 


\section{Evaluation}

Kernfrage: Inwieweit berücksichtigt die Evaluation des Programms Empowerment-Aspekte?

Der letzte Schritt des „parallel-tracking“ ist die Evaluation der Ergebnisse im Hinblick auf das Präventionsprogramm und auf Empowerment. Empowerment auf Gemeindeebene ist häufig ein langer Vorgang, und wenn es nur im Rahmen der Ergebnisevaluation bewertet wird, kann das, was erreicht wurde, durch den bei Evaluationen üblichen, relativ engen Zeitrahmen übersehen werden. Die Evaluation von Empowerment als dynamischem Prozess ist daher immer der Evaluation eines konkreten Empowerment-Ergebnisses vorzuziehen.

Um das Beispiel des Präventionsprogramms für das metabolische Syndrom wieder aufzugreifen: Hier könnte die Evaluation von Empowerment folgende Indikatoren in Anlehnung an die gesetzten Ziele verwenden:

- Die Anzahl der Personen, die sich durch das Programm bei den Sportangeboten und -einrichtungen angemeldet haben.

- Die Anzahl der aktiven Walking-Gruppen, die sich während des Programms eigenständig gegründet haben.

- Die Anzahl der abgeschlossenen Seminare und die Anzahl der Personen, die sich für weiterführende Kurse und Diätklassen angemeldet haben.

Indem Empowerment als Prozess gemessen wird, ist es möglich, die wechselseitige Beeinflussung von Kapazitäten, Fähigkeiten und Ressourcen sowohl auf individueller als auch organisatorischer bzw. Gemeinde-Ebene während des gesamten Programms zu erfassen. Die Evaluation des Programms kann gleichzeitig eine ermächtigende („empowernde“) Erfahrung für die Gemeinde sein. Daher sollte sie idealerweise durch die Zielgruppe bzw. Gemeinde selbst vorgenommen werden, bzw. diese sehr stark einbeziehen. Auf individueller Ebene ist Empowerment häufig unmittelbarer zu messen, z. B. durch eine Steigerung von Selbstbewusstsein und Vertrauen. Bei der Evaluation sollte dennoch nicht der individuelle Fokus im Vordergrund stehen, sondern die Entwicklung auf kollektiver Ebene, denn sie zeigt die Fähigkeit auf, Empowerment innerhalb der Gruppe auszubilden.
Um das Empowerment und die Kapazität auf Ebene einer Gruppe oder Gemeinde als Prozess zu messen, bietet sich eine systematische Vorgehensweise an, bei der Mitglieder der Gruppe oder Gemeinde ihren Stand bei den neun Kapazitätsbereichen (s.o.) selber bewerten. Die Selbsteinschätzung wird durch ein Spinnennetz-Diagramm visualisiert (s. Laverack G, „Messung, Bewertung und strategische Weiterentwicklung von Gemeindekapazität und -empowerment: Vorstellen eines qualitativen Instruments“ in dieser Ausgabe, S.764). In einem Gesundheitsförderungs-Programm mit begrenzten Ressourcen hat dies den Vorteil, dass die benötigte Zeit und die benötigten Ressourcen auf ein Minimum reduziert werden, da der Fokus auf relevante Bereiche gelegt wird.

Der Autor ist an einem Erfahrungsaustausch mit Forschern oder Programmleitern aus Deutschland interessiert, die ähnliche Ansätze zur Ausbildung und Messung von Empowerment auf Gemeindeebene verwenden.

\section{Danksagung}

Der Autor dankt Frau PD Dr. Julika Loss für ihre wertvollen Anmerkungen, die zur Erstellung dieses Artikels beigetragen haben, und ihre Übersetzung ins Deutsche.

\section{Literatur}

1 Laverack $G$, Labonte $R$. A planning framework for the accommodation of community empowerment goals within health promotion programming. Health Policy Planning 2000; 15: 255-262

2 Laverack G. Health Promotion Practice: Power and Empowerment. Sage Publications, London; 2004

3 Laverack G. Health Promotion Practice: building empowered communities. Open University Press, London; 2007

4 Max Rubner-Institut (Bundesforschungsinstitut für Ernährung und Lebensmittel, Hrsg. Nationale Verzehrsstudie II. Ergebnisbericht, Teil 1. Karlsruhe; 2008

5 Ewles L, Simnett I. Promoting health. A practical guide. 5. Auflage. Bailliere Tindall, London; 2003

6 Laverack G. Public Health: Power, Empowerment and Professional Practice. Palgrave Macmillan, London; 2005 